\title{
Perturbative expansions of Rényi relative divergences and holography
}

\author{
Tomonori Ugajin \\ Okinawa Institute of Science and Technology, \\ Tancha, Kunigami gun, Onna son, Okinawa 1919-1, Japan \\ E-mail: tomonori.ugajin@yukawa.kyoto-u.ac.jp
}

ABSTRACT: In this paper, we develop a novel way to perturbatively calculate Rényi relative divergences $D_{\gamma}(\rho \| \sigma)=\operatorname{tr} \rho^{\gamma} \sigma^{1-\gamma}$ and related quantities without using replica trick as well as analytic continuation. We explicitly determine the form of the perturbative term at any order by an integral along the modular flow of the unperturbed state. By applying the prescription to a class of reduced density matrices in conformal field theory, we find that the second order term of certain linear combination of the divergences has a holographic expression in terms of bulk symplectic form, which is a one parameter generalization of the statement "Fisher information = Bulk canonical energy".

KeYwords: AdS-CFT Correspondence, Gauge-gravity correspondence, Holography and condensed matter physics (AdS/CMT)

ArXiv EPrint: 1812.01135 


\section{Contents}

1 Introduction 1

2 New expansion formula using the resolvent trick 3

3 Some explicit checks $\quad 4$

$3.1 \quad$ First order term $T_{\gamma}^{(1)}(\delta \rho) \quad 5$

3.2 Second order term $T_{\gamma}^{(2)}(\delta \rho) \quad 5$

3.2.1 Checks 5

4 Expressions of perturbative terms in terms of the vacuum modular flow 6

$\begin{array}{lll}4.1 & \text { Doing the Fourier transformation } & 7\end{array}$

4.2 Choice of the integration contour: the quadratic $n=2$ term 8

4.3 Contour choice: $n \geq 3$ terms 9

5 Applications to conformal field theory $\quad 10$

$\begin{array}{lll}5.1 \text { Set up } & 10\end{array}$

5.2 The perturbative expression of $T_{\gamma}(\rho) \quad 11$

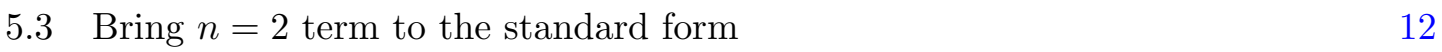

6 Expansion of Petz's quasi entropy $D_{\gamma}(\rho \| \sigma) \quad 12$

6.1 Expressing $X_{\gamma}(\delta \rho)$ and $Y_{\gamma}(\delta \rho)$ by modular flow integrals $\quad 13$

6.2 Holographic expressions of $X_{\gamma}(\delta \rho)$ and $Y_{\gamma}(\delta \rho) \quad 14$

$\begin{array}{lll}6.2 .1 & \text { Set up } & 14\end{array}$

$\begin{array}{lll}\text { 6.2.2 Holographic rewritings } & 15\end{array}$

$\begin{array}{lll}7 & \text { Conclusions } & 17\end{array}$

$\begin{array}{ll}\text { A The calculation of } \mathcal{K}_{\gamma}^{(n)}\left(s_{1}, \cdots s_{n-1}\right) & 17\end{array}$

$\begin{array}{ll}\text { B Fixing the contour of } n=2 \text { term } & 19\end{array}$

$\begin{array}{lr}\text { C Simplifying } T_{\gamma}^{(2)}(\delta \rho) & 20\end{array}$

$\begin{array}{ll}\text { D Direct Fourier transformation } & 22\end{array}$

$\begin{array}{ll}\text { E Details of the holographic rewriting } & 22\end{array}$ 


\section{Introduction}

The concept of entanglement is one of the keys to understand how holography works. This idea is supported by the Ryu Takayanagi formula [1, 2] and its covariant generalization [3], which relate the area of particular extremal surfaces in the bulk, to the entanglement entropies in the dual conformal field theory (CFT). As a concrete and quantitative application of this entanglement vs gravity program, recently it has been shown that bulk gravitational dynamics can be read off from the entanglement structure of states in the dual CFT.

In this line of developments, it was initially observed that so called first law of entanglement [4] is related to the linearized Einstein equations in the bulk [5, 6]. Consider starting from the vacuum reduced density matrix $\rho_{0}$ and making it excited slightly $\rho_{0} \rightarrow \rho_{0}+\delta \rho$ in a CFT. The change of the entanglement entropy $\delta S$ obeys first law of entanglement, $\delta S=\operatorname{tr}\left[K \delta_{0}\right]$, where $K=-\log \rho_{0}$ is called modular Hamiltonian of $\rho_{0}$. For the subsystems of special type, the vacuum modular Hamiltonian has a local expression given by an integral of energy density over the subsystem. There is a natural bulk counterpart of the vacuum modular Hamiltonian, namely, the generator of time translation of a topological black hole with hyperbolic horizon, whose Bekenstein Hawking entropy gives the CFT vacuum entanglement entropy [7]. The first law of entanglement is related to the first law of thermodynamics applied to the topological black hole, and this enabled us to read off the linearized equations of motion.

Recently this nice story at the first order in the perturbation $\delta \rho$ has been generalized to the quadratic order. It was noticed that, in CFT the second order change of the entanglement entropy can be concisely summarized as an integral of correlation functions along the flow generated by the vacuum modular Hamiltonian $K=-\log \rho_{0}$ on the subsystem [8-10]. This was further extended to arbitrarily order in $\delta \rho$ and some technical issue was pointed out [11]. It was also recognized that by rewriting the CFT answer in terms of bulk variables, we naturally identify it with bulk canonical energy [12], which was first found holographically in [13]. This makes it possible to read off the bulk equations of motion beyond the linearized level.

Given these developments, it is now natural to generalize this story to other quantum information theoretic quantities. In particular we would like to find such a quantity which admits a nice perturbative expansion in CFT and has a dual holographic expression. Natural candidates having these properties are those involving powers of reduced density matrices, for example tr $\rho^{\gamma}$ which is related to Rényi entropy.

Conventionally, a Rényi type quantity, like $\operatorname{tr} \rho^{\gamma}$ has been computed by replica trick. In this trick, we first regard the Rényi index to be a positive integer $\gamma=n$, and represent the quantity as a path integral on a branched space $\Sigma_{n}$ which is prepared by gluing $n$ copies of the original space with cuts along the subsystems. After the computation of the path integral, we then analytically continue the integer $n$ to arbitrarily number $\gamma$. However, this trick has several disadvantages, even when we compute the quantity perturbatively. First of all, the analytic continuation is usually difficult to perform. For example, when we 
perturbatively expand $\operatorname{tr} \rho^{n}$ for $\rho=\rho_{0}+\delta \rho$, at quadratic order we encounter following sum

$$
\sum_{k, m} \operatorname{tr}\left[\rho_{0}^{k-1} \delta \rho \rho_{0}^{m-k-1} \delta \rho \rho_{0}^{n-m}\right] .
$$

In order to analytically continue it in $n$ we first need to perform this sum to get a closed expression. Although for special cases we can do this, in general it is difficult. In addition to this, we do not know how to do analogous sums for the cubic term and higher. Second, there are ambiguities in the analytic continuations. According to the Carson's theorem, we need to correctly specify the behavior of $\operatorname{tr} \rho^{n}$ on certain region of the complex $n$ plane, in order to fix the ambiguities.

In order to overcome these difficulties, in this paper we would like to develop a new way to perturbatively calculate Rényi type quantities without using replica trick, and analytic continuation. The idea we employ is simple, namely writing $\operatorname{tr} \rho^{\gamma}$ by a contour integral,

$$
\operatorname{tr} \rho^{\gamma}=\int_{C} \frac{d z}{2 \pi i} z^{\gamma} \operatorname{tr} \frac{1}{z-\rho}
$$

where the contour $C$ is chosen so that it includes all the poles of the integrand, but avoid the contribution of the branch cut coming from $z^{\gamma}$. We refer to $[14,15]$ for discussions on the representation. By expanding the denominator of the integrand for perturbative states $\rho=\rho_{0}+\delta \rho$, we can systematically write each term of the perturbative expansion by an integral along the modular flow of the reference state $\rho_{0}$. If we apply this expansion for a class of perturbative excited states from vacuum in a $d$ dimensional CFT, we can write each term as an integral of a correlation function $\langle\cdots\rangle_{\Sigma_{\gamma}}$ on the branched space $\Sigma_{\gamma}=S_{\gamma}^{1} \times H^{d-1}$ along the modular flow generated by $\rho_{0}$. Here, $S_{\gamma}^{1}$ denotes the Euclidean time circle with $2 \pi \gamma$ periodicity, and $H^{d-1}$ is $d-1$ dimensional hyperbolic space. Of course, the CFT correlation functions on $\Sigma_{\gamma}$ are difficult to calculate when $d>2$, as the branched space $\Sigma_{\gamma}$ is not conformally related to d dimensional flat space, and even two point functions are highly theory dependent ones.

However, by the same trick, we can similarly expand the Petz's quasi entropy [16] defined by,

$$
D_{\gamma}(\rho \| \sigma)=\operatorname{tr} \rho^{\gamma} \sigma^{1-\gamma} .
$$

This quantity can be regarded as a one parameter generalization of relative entropy,

$$
\left.\frac{d}{d \gamma} D_{\gamma}(\rho \| \sigma)\right|_{\gamma=1}=S(\rho \| \sigma)=\operatorname{tr} \rho \log \rho-\operatorname{tr} \rho \log \sigma .
$$

We also refer to recent studies on Rényi generalizations of relative entropy [17-22] as well as perturbative calculations of relative entropy [11, 23-29]. One notable feature of this Rényi relative divergence is that, each term of its perturbative expansion involves a correlator on the regular space $\Sigma_{1}$ which is conformally related to flat space. This implies that the first few terms of the expansion are almost fixed by conformal symmetry, and independent of the CFT we consider. In particular, this property enables us to holographically write the quadratic terms of certain linear combinations of $D_{\gamma}(\rho \| \sigma)$ which we will denote by $X_{\gamma}(\delta \rho), Y_{\gamma}(\delta \rho)$, in terms of bulk symplectic form, without the details of the bulk 
to boundary dictionary. This generalizes the statement "quantum fisher information = bulk canonical energy". See also $[30,31]$ for recent discussions on bulk symplectic form.

This paper is organized as follows. In section 2 , we explain how to expand $T_{\gamma}(\rho)=\operatorname{tr} \rho^{\gamma}$ using the formula (1.2). We first derive expressions of the perturbative terms as integrals with respect to the entanglement spectrum of the unperturbed state. In section 3, we check these expressions against known results. In section 4 we express each term of the perturbative expansion as an integral along the modular flow of the unperturbed state by Fourier transforming the spectral representation of the kernel derived in section 2. In section 5 we apply the formalism to reduced density matrices in conformal field theory, and write these perturbative terms in terms of correlation functions in CFT. In section 6, we discuss a similar expansion of Petz's quasi entropy and derive a holographic expression of the second order term.

\section{New expansion formula using the resolvent trick}

In the first few sections we focus on the Rényi type quantity

$$
T_{\gamma}(\rho)=\operatorname{tr} \rho^{\gamma} .
$$

In the discussions we do not assume the index $\gamma$ to be an positive integer $\gamma \in \mathbb{Z}_{+}$, where one can use the replica trick. Although we will apply the prescription developing here to conformal field theory, the discussions in this section and the next few ones are applicable for any density matrix of any theory.

When the density matrix $\rho$ is sufficiently close to the reference state $\rho_{0}$, ie $\rho=\rho_{0}+\delta \rho$, we can expand $T_{\gamma}(\rho)$ by a power series of $\delta \rho$,

$$
T_{\gamma}(\rho)=T_{\gamma}\left(\rho_{0}\right)+\sum_{n=0}^{\infty} T_{\gamma}^{(n)}(\delta \rho),
$$

and decompose each term in the perturbative expansion by the spectra of the reference state $\rho_{0}$. Let us first do this.

We begin the discussion by first writing $T_{\gamma}(\rho)$ using the resolvent of $\rho$,

$$
\operatorname{tr} \rho^{\gamma}=\int_{C} \frac{d z}{2 \pi i} z^{\gamma} \operatorname{tr} \frac{1}{z-\rho},
$$

where the contour $C$ is encircling the interval $\left[\rho_{\min }, 1\right]$ in the $z$ plane, but not $z=0$, so that it picks up all contributions of eigenvalues of $\rho . \rho_{\min }$ is the smallest eigenvalue of the density matrix $\rho$. (See figure 1.) When $\rho$ is a reduced density matrix of a quantum field theory, we need to put a UV cut off $\varepsilon$ so that the density matrix $\rho$ has a minimum eigenvalue, then after the calculation we send $\varepsilon \rightarrow 0$. We will explicitly see that only the unperturbed term $T_{\gamma}\left(\rho_{0}\right)$ depends on the UV cutoff and rests do not. Therefore we can uniquely fix the form of $T_{\gamma}^{(n)}(\delta \rho), n \geq 1$.

When $\rho=\rho_{0}+\delta \rho$ the resolvent can be easily expanded,

$$
\frac{1}{z-\rho}=\sum_{n=0}^{\infty} R_{n}(\delta \rho) \quad R_{n}(\delta \rho)=\left(\frac{1}{\left(z-\rho_{0}\right)} \delta \rho\right)^{n} \frac{1}{\left(z-\rho_{0}\right)}
$$




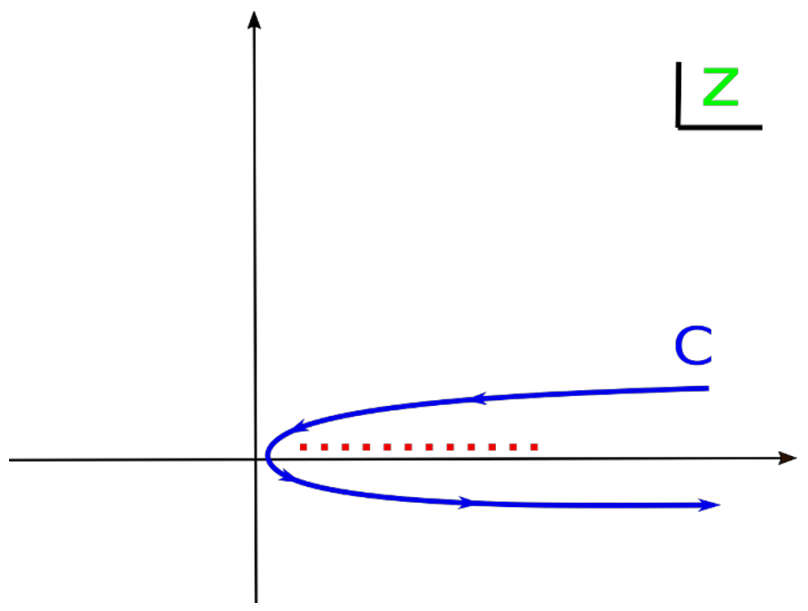

Figure 1. The contour $C$ of the integral (2.3)(blue line). Red dots are the poles of the meromorphic function $f(z)=\operatorname{tr} \frac{1}{z-\rho}$. These poles are in the segment $0<\operatorname{Re} z<1$.

By inserting the complete set of eigenstates $\left|\omega_{i}\right\rangle$ of the reference state $\rho_{0}$,

$$
\int d \omega_{i}\left|\omega_{i}\right\rangle\left\langle\omega_{i}\left|=1, \quad \rho_{0}\right| \omega_{i}\right\rangle=e^{-2 \pi \omega_{i}}\left|\omega_{i}\right\rangle,
$$

to the left of $i$-th term, taking trace, and evaluating $1 /\left(z-\rho_{0}\right)$ from the left, we have,

$$
\begin{aligned}
\operatorname{tr}\left[R_{n}(\delta \rho)\right] & =\int \prod_{i=1}^{n} d \omega_{i} \prod_{i=1}^{n-1} \frac{1}{z-e^{-2 \pi \omega_{i}}} \prod_{k=1}^{n-1}\left\langle\omega_{k}|\delta \rho| \omega_{k+1}\right\rangle\left\langle\omega_{n}\left|\frac{1}{\left(z-\rho_{0}\right)} \delta \rho \frac{1}{\left(z-\rho_{0}\right)}\right| \omega_{1}\right\rangle \\
& =\int \prod_{i=1}^{n} d \omega_{i} \frac{1}{\left(z-e^{-2 \pi \omega_{1}}\right)^{2}} \prod_{i=2}^{n} \frac{1}{z-e^{-2 \pi \omega_{i}}} \prod_{k=1}^{n}\left\langle\omega_{k}|\delta \rho| \omega_{k+1}\right\rangle
\end{aligned}
$$

in the last term, $\omega_{n+1} \equiv \omega_{1}$ is understood.

In summary, here we expanded $T_{\gamma}(\rho)$ with respect to $\delta \rho$, as in $(2.2)$, and saw that the $n$th order term of the expansion $T_{\gamma}^{(n)}(\delta \rho)$ is given by

$$
T_{\gamma}^{(n)}(\delta \rho)=\int \prod_{i=1}^{n} d \omega_{i}\left[\int_{C} \frac{d z}{2 \pi i} z^{\gamma} \frac{1}{\left(z-e^{-2 \pi \omega_{1}}\right)^{2}} \prod_{i=2}^{n} \frac{1}{z-e^{-2 \pi \omega_{i}}}\right] \prod_{k=1}^{n}\left\langle\omega_{k}|\delta \rho| \omega_{k+1}\right\rangle .
$$

By defining the kernel function,

$$
K^{(n)}\left(\omega_{1}, \cdots \omega_{n}\right) \equiv \int_{C} \frac{d z}{2 \pi i} \frac{z^{\gamma}}{\left(z-e^{-2 \pi \omega_{1}}\right)^{2}} \prod_{i=2}^{n} \frac{1}{z-e^{-2 \pi \omega_{i}}},
$$

we write,

$$
T_{\gamma}^{(n)}(\delta \rho)=\int \prod_{i=1}^{n} d \omega_{i} K^{(n)}\left(\omega_{1}, \cdots \omega_{n}\right) \prod_{k=1}^{n}\left\langle\omega_{k}|\delta \rho| \omega_{k+1}\right\rangle .
$$

\section{Some explicit checks}

We have obtained the perturbative expansion using the spectrum of the reference state $\rho_{0}$. To get some insights, in this section we explicitly write down first few terms of the expansion and check them against known results. 


\subsection{First order term $T_{\gamma}^{(1)}(\delta \rho)$}

The first order term of the series is given by

$$
\begin{aligned}
T_{\gamma}^{(1)}(\delta \rho) & =\int d \omega\langle\omega|\delta \rho| \omega\rangle \int_{C} \frac{d z}{2 \pi i} \frac{z^{\gamma}}{\left(z-e^{-2 \pi \omega}\right)^{2}} \\
& =\gamma \operatorname{tr}\left[\rho_{0}^{\gamma-1} \delta \rho\right]
\end{aligned}
$$

as it should be.

\subsection{Second order term $T_{\gamma}^{(2)}(\delta \rho)$}

Let us move on to the second order term $T_{\gamma}^{(2)}(\delta \rho)$. It is given by

$$
T_{\gamma}^{(2)}(\delta \rho)=\int d \omega d \omega^{\prime}\left\langle\omega|\delta \rho| \omega^{\prime}\right\rangle\left\langle\omega^{\prime}|\delta \rho| \omega\right\rangle K\left(\omega, \omega^{\prime}\right)
$$

Precise form of $K\left(\omega, \omega^{\prime}\right)$ can be derived by the contour integral,

$$
\begin{aligned}
K\left(\omega, \omega^{\prime}\right) & =\int_{C} \frac{d z}{2 \pi i} \frac{z^{\gamma}}{\left(z-e^{-2 \pi \omega}\right)^{2}\left(z-e^{-2 \pi \omega^{\prime}}\right)} \\
& =\frac{1}{\left(e^{-2 \pi \omega^{\prime}}-e^{-2 \pi \omega}\right)^{2}}\left[(\gamma-1) e^{-2 \pi \gamma \omega}+e^{-2 \pi \gamma \omega^{\prime}}-\gamma e^{-2 \pi(\gamma-1) \omega} e^{-2 \pi \omega^{\prime}}\right] .
\end{aligned}
$$

\subsubsection{Checks}

$\gamma=\boldsymbol{n} \in \mathbb{Z}_{+}$. When the index $\gamma$ is a positive integer, the kernel $K_{\gamma}\left(\omega, \omega^{\prime}\right)$ is decomposed into the sum,

$$
K\left(\omega, \omega^{\prime}\right)=\left[\sum_{l=0}^{\gamma-2}((\gamma-1)-l)\left(e^{-2 \pi \omega}\right)^{\gamma-l}\left(e^{-2 \pi \omega^{\prime}}\right)^{l}\right] .
$$

Plugging this into (3.2) and undoing the spectral decomposition, we recover the obvious expansion (1.1) which we frequently encounter in replica calculations. The kernel avoids the difficulties of replica trick, by automatically doing the summation as well as analytic continuation in $n$.

The von Neumann entropy limit. $T_{\gamma}(\rho)$ is related to the von Neumann entropy $S(\rho)$ by

$$
S(\rho)=-\operatorname{tr} \rho \log \rho=\left.\frac{\partial}{\partial \gamma} T_{\gamma}(\rho)\right|_{\gamma=1}
$$

From (3.3) we derive the kernel for the quadratic part of the von Neumann entropy,

$$
\left.\frac{\partial K_{\gamma}}{\partial \gamma}\right|_{\gamma=1}=\frac{e^{2 \pi \omega}}{\left(1-e^{2 \pi\left(\omega-\omega^{\prime}\right)}\right)}\left[\left(e^{-2 \pi \omega}-e^{-2 \pi \omega^{\prime}}\right)+2 \pi\left(\omega-\omega^{\prime}\right) e^{-2 \pi \omega^{\prime}}\right] .
$$

In [8], a perturbative expansion of the von Neumann entropy $S\left(\rho_{0}+\delta \rho\right)$ was discussed, by expanding the modular Hamiltonian $K_{\rho}=-\rho_{0}+\delta \rho$ using the identity,

$$
\log \rho=\int_{0}^{\infty} d \beta\left(\frac{1}{\rho+\beta}-\frac{1}{\beta+1}\right),
$$

the result of the quadratic order kernel in [8] agrees with (3.6). 


\section{Expressions of perturbative terms in terms of the vacuum modular flow}

The $\omega$ integrals in the right hand side of (2.9) are of course hard to perform, as we do not know precise form of the eigenvalue distribution of $\rho_{0}$. To proceed, we now express each term of the perturbative series $T_{\gamma}^{(n)}(\delta \rho)$ as an integral along the modular flow of $\rho_{0}$, by Fourier transforming the kernel $\mathcal{K}_{\gamma}^{(n)}\left(\omega_{1}, \cdots \omega_{n}\right)$.

This process is very analogous to the case of the von Neumann entropy perturbation done in [8] for quadratic order term and generalized to higher order terms in [11]. It is convenient to introduce the rescaled kernel, defined by

$$
\begin{aligned}
\mathcal{K}_{\gamma}^{(n)}\left(\omega_{1}, \cdots \omega_{n}\right) & \equiv e^{2 \pi \gamma \omega_{1}-2 \pi \sum_{k=1}^{n} \omega_{k}} K^{(n)}\left(\omega_{1}, \cdots \omega_{n}\right) \\
& =\int_{C} \frac{d z}{2 \pi i} z^{\gamma} \frac{e^{2 \pi(\gamma-1) \omega_{1}}}{\left(z-e^{-2 \pi \omega_{1}}\right)^{2}} \prod_{i=2}^{n} \frac{e^{-2 \pi \omega_{i}}}{z-e^{-2 \pi \omega_{i}}} .
\end{aligned}
$$

Using this function, we get

$$
\begin{aligned}
T_{\gamma}^{(n)}(\delta \rho) & =\int \prod_{i=1}^{n} d \omega_{i} K^{(n)}\left(\omega_{1}, \cdots \omega_{n}\right) \prod_{k=1}^{n}\left\langle\omega_{k}|\delta \rho| \omega_{k+1}\right\rangle \\
& =\int \prod_{i=1}^{n} d \omega_{i} e^{-2 \pi \gamma \omega_{1}+2 \pi \sum_{k=1}^{n} \omega_{k}} \mathcal{K}_{\gamma}^{(n)}\left(\omega_{1}, \cdots \omega_{n}\right) \prod_{k=1}^{n}\left\langle\omega_{k}|\delta \rho| \omega_{k+1}\right\rangle \\
& =\int \prod_{i=1}^{n} d \omega_{i} \mathcal{K}_{n}^{\gamma}\left(\omega_{1}, \cdots \omega_{n}\right)\left\langle\omega_{1}\left|e^{-2 \pi \gamma K} \delta \tilde{\rho}\right| \omega_{2}\right\rangle \prod_{k=1}^{n}\left\langle\omega_{k}|\tilde{\delta} \rho| \omega_{k+1}\right\rangle,
\end{aligned}
$$

where $2 \pi K=-\log \rho_{0}$ is the modular Hamiltonian of $\rho_{0}$, and $\tilde{\delta} \rho=e^{\pi K} \delta \rho e^{\pi K}$. It can be easily shown that the new kernel $\mathcal{K}_{\gamma}^{(n)}\left(\omega_{1}, \cdots \omega_{n}\right)$ is invariant under the shifts $\omega_{i} \rightarrow \omega_{i}+\alpha$,

$$
\mathcal{K}_{\gamma}^{(n)}\left(\omega_{1}+\alpha, \cdots \omega_{n}+\alpha\right)=\mathcal{K}_{\gamma}^{(n)}\left(\omega_{1}, \cdots \omega_{n}\right)
$$

So if we change the variables to $\left\{a_{i}, b\right\}$,

$$
a_{i}=\omega_{i}-\omega_{i+1}, i=1 \cdots n-1 \quad b=\sum_{i=1}^{n} \omega_{i}
$$

$\mathcal{K}_{\gamma}^{(n)}\left(\omega_{1}, \cdots \omega_{n}\right)$ only depends on $n-1$ variables $\left\{a_{i}\right\}_{i=1 \cdots n-1}$,

$$
\mathcal{K}_{\gamma}^{(n)}\left(\omega_{1}, \cdots \omega_{n}\right)=\mathcal{K}_{\gamma}^{(n)}\left(a_{1}, a_{2}, \cdots a_{n-1}\right)
$$

Thanks to this property $\mathcal{K}_{\gamma}^{(n)}\left(\omega_{1}, \cdots \omega_{n}\right)$ has a nice Fourier transformation,

$$
\mathcal{K}_{\gamma}^{(n)}\left(\omega_{1}, \cdots \omega_{n}\right)=\int_{C} d s_{1} \cdots d s_{n-1} e^{i \sum_{k=1}^{n-1} s_{k} a_{k}} \mathcal{K}_{\gamma}^{(n)}\left(s_{1}, \cdots s_{n-1}\right),
$$

$\left\{s_{i}\right\}_{i=1 \cdots n-1}$ are variables dual to the spectrum of $\rho_{0}$, therefore they have a geometric interpretation, ie, they are parameterizing the modular flow of $\rho_{0}$. Also, as we will see 
later, we need to properly choose the integration contours $C$ in order for the Fourier transformation (4.7) to correctly reproduce the kernel $\mathcal{K}_{\gamma}^{(n)}\left(\omega_{1}, \cdots \omega_{n}\right)$.

Using this and undoing the spectral decompositions (2.5), we can write $T_{\gamma}^{(n)}(\delta \rho)$ as an integral of real time $\left\{s_{i}\right\}$ variables,

$$
\begin{aligned}
T_{\gamma}^{(n)}(\delta \rho) & =\int \prod_{i=1}^{n} d \omega_{i} \mathcal{K}_{\gamma}^{(n)}\left(\omega_{1}, \cdots \omega_{n}\right)\left\langle\omega_{1}\left|e^{-2 \pi \gamma K} \delta \tilde{\rho}\right| \omega_{2}\right\rangle \prod_{k=1}^{n}\left\langle\omega_{k}|\tilde{\delta} \rho| \omega_{k+1}\right\rangle \\
& =\int_{C} d s_{1} \cdots d s_{n-1} \mathcal{K}_{\gamma}^{(n)}\left(s_{1}, \cdots s_{n-1}\right) \operatorname{tr}\left[e^{-2 \pi \gamma K} \prod_{k=1}^{n-1} e^{i K s_{k}} \tilde{\delta} \rho e^{-i K s_{k}} \tilde{\delta} \rho\right]
\end{aligned}
$$

In the actual CFT computations, this undoing is a bit tricky, and needed special cares. We will discuss on this in the latter sections.

\subsection{Doing the Fourier transformation}

Let us first specify the form of the real time kernel $\mathcal{K}_{\gamma}^{(n)}\left(s_{1}, \cdots s_{n-1}\right)$. The task is doing the inverse Fourier transformation,

$$
\mathcal{K}_{\gamma}^{(n)}\left(s_{1}, \cdots s_{n-1}\right)=\int \frac{d a_{1} \cdots d a_{n-1}}{(2 \pi)^{n-1}} e^{-i \sum_{k=1}^{n-1} s_{k} a_{k}} \mathcal{K}_{\gamma}^{(n)}\left(a_{1}, \cdots a_{n-1}\right) .
$$

The trick we use is very similar to the one developed in our previous paper [11]. By inserting a delta function,

$$
\delta(q)=\frac{1}{2 \pi} \int d b e^{-i q b}
$$

we can disentangle the multiple integral to a product of integrals of single variables $\left\{\omega_{i}\right\}$,

$$
\begin{aligned}
\delta(q) \mathcal{K}_{\gamma}^{(n)}\left(s_{1}, \cdots s_{n-1}\right) & =\frac{1}{(2 \pi)^{n}} \int d b e^{-i q b} \int d a_{1} \cdots d a_{n-1} e^{-i \sum_{k=1}^{n-1} s_{k} a_{k}} \mathcal{K}_{\gamma}^{(n)}\left(a_{1}, \cdots a_{n-1}\right) \\
& =\frac{n}{(2 \pi)^{n}} \int d \omega_{1} \cdots d \omega_{n} e^{-i q b} e^{-i \sum_{k=1}^{n-1} s_{k} a_{k}} \mathcal{K}_{\gamma}^{(n)}\left(\omega_{1}, \cdots \omega_{n}\right),
\end{aligned}
$$

in the second line we used the relations (4.5).

Now the integral is

$$
\begin{aligned}
\delta(q) \mathcal{K}_{\gamma}^{(n)}\left(s_{1}, \cdots s_{n-1}\right)= & \frac{n}{(2 \pi)^{n}} \int d \omega_{1} \cdots d \omega_{n} e^{-i q b} e^{-i \sum_{k=1}^{n-1} s_{k} a_{k}} \mathcal{K}_{\gamma}^{(n)}\left(\omega_{1}, \cdots \omega_{n}\right) \\
= & \frac{n}{(2 \pi)^{n}} \int_{C} \frac{d z}{2 \pi i} z^{\gamma} \int d \omega_{1} \frac{e^{-\omega_{1}\left[-2 \pi(\gamma-1)+i\left(s_{1}+q\right)\right]}}{\left(z-e^{-2 \pi \omega_{1}}\right)^{2}} \\
& \times \prod_{i=2}^{n-1} \int d \omega_{i} \frac{e^{-\omega_{i}\left[2 \pi+\left(s_{k}-s_{k-1}+q\right) i\right]}}{z-e^{-2 \pi \omega_{i}}} \\
& \times \int d \omega_{n} \frac{e^{-\omega_{n}\left[2 \pi-\left(s_{n-1}-q\right) i\right]}}{z-e^{-2 \pi \omega_{n}}} \\
\equiv & \frac{n}{(2 \pi)^{n}} \int_{C} \frac{d z}{2 \pi i} J(z) .
\end{aligned}
$$


The strategy to compute this complicated integral is first compute each $\omega_{i}$ integral, and express $J(z)$ as a function of modular times $\left\{s_{i}\right\}_{i=1 \cdots n-1}$. We then perform the $z$ integral by choosing the contour along the real axis,

$$
\delta(q) \mathcal{K}_{\gamma}^{(n)}\left(s_{1}, \cdots s_{n-1}\right)=\frac{n}{(2 \pi)^{n}} \int_{0}^{\infty} \frac{d \beta}{2 \pi i}(J(\beta-i \epsilon)-J(\beta+i \epsilon)), \quad \epsilon \rightarrow 0_{+} .
$$

The details of the calculation can be found in appendix A and here we only present the final result for the kernel $\mathcal{K}_{\gamma}^{(n)}\left(s_{1}, \cdots s_{n-1}\right)$,

$$
\mathcal{K}_{\gamma}^{(n)}\left(s_{1}, \cdots s_{n-1}\right)=\frac{i}{8 \pi^{2}}\left(\frac{-i}{4 \pi}\right)^{n-2} \frac{\left(s_{1}+2 \pi i \gamma\right) \sin \pi \gamma}{\sinh \left(\frac{s_{1}+2 \pi i \gamma}{2}\right) \prod_{k=2}^{n-1} \sinh \left(\frac{s_{k}-s_{k-1}}{2}\right) \sinh \left(\frac{s_{n-1}}{2}\right)}
$$

\subsection{Choice of the integration contour: the quadratic $n=2$ term}

In the previous subsection we derived the expression (4.14) of the real time kernel $\mathcal{K}_{\gamma}^{(n)}\left(s_{1}, \cdots s_{n-1}\right)$. In order to complete the discussion we need to properly fix the contour of the real time integrals $C$ in (4.8). We can do so by demanding the Fourier transformation can be correctly reversed,

$$
\mathcal{K}_{\gamma}^{(n)}\left(\omega_{1}, \cdots, \omega_{n}\right)=\int_{C_{k}} \prod_{k=1}^{n-1} d s_{k} e^{i \sum_{k=1}^{n-1} s_{k} a_{k}} \mathcal{K}_{\gamma}^{(n)}\left(s_{1}, \cdots s_{n-1}\right) .
$$

We first consider the contour of quadratic $n=2$ term,

$$
\int_{C_{s}} d s \mathcal{K}_{\gamma}^{(2)}(s) e^{i a s}=\frac{i \sin \pi \gamma}{8 \pi^{2}} \int_{C_{s}} d s \frac{s+2 \pi i \gamma}{\sinh \frac{s}{2} \sinh \frac{s+2 \pi i \gamma}{2}} e^{i a s}
$$

which is a bit tricky compared to higher order terms. When $a>0$ we close the contour on the upper half plane.

The real time kernel $\mathcal{K}_{\gamma}^{(2)}(s)$ has two types of poles.

$$
s_{1}^{n}=2 \pi i n, \quad s_{2}^{k}=2 \pi i(k-\gamma), \quad n, k \in \mathbb{Z}, \quad k \neq 0 .
$$

We can easily see that if one choose the contour $C_{s}$ which contains $s_{1}^{n}, n \geq 0$, and $s_{2}^{k}, k \geq 1$ (as in figure 2), then the Fourier transformation is correctly reversed,

$$
\mathcal{K}_{\gamma}^{(n)}(a)=\int_{C_{s}} d s \mathcal{K}_{\gamma}^{(2)}(s) e^{i a s}
$$

Again we explicitly check this in appendix B.

It is useful to write the integral as follows. Since we can write the integrand,

$$
\frac{(s+2 \pi i \gamma) \sin \pi \gamma}{\sinh \frac{s}{2} \sinh \frac{s+2 \pi i \gamma}{2}}=\frac{s+2 \pi i \gamma}{1-e^{-s}}-\frac{s+2 \pi i \gamma}{1-e^{-(s+2 \pi i \gamma)}}
$$

then, the contour integral is naturally split into two parts,

$$
\begin{aligned}
\int_{C_{s}} d s \frac{(s+2 \pi i \gamma) \sin \pi \gamma}{\sinh \frac{s}{2} \sinh \frac{s+2 \pi i \gamma}{2}} G(s)= & \int_{-\infty-i \epsilon}^{\infty-i \epsilon} d s\left[\frac{s+2 \pi i \gamma}{1-e^{-s}}\right] G(s) \\
& -\int_{-\infty-2 \pi i(\gamma-\epsilon)}^{\infty-2 \pi i(\gamma-\epsilon)} d s\left[\frac{s+2 \pi i \gamma}{1-e^{-(s+2 \pi i \gamma)}}\right] G(s)
\end{aligned}
$$

for any function $G(s)$ which is holomorphic on the strip $-2 \pi \gamma<\operatorname{Im} s<0$, when $\gamma>0$. 


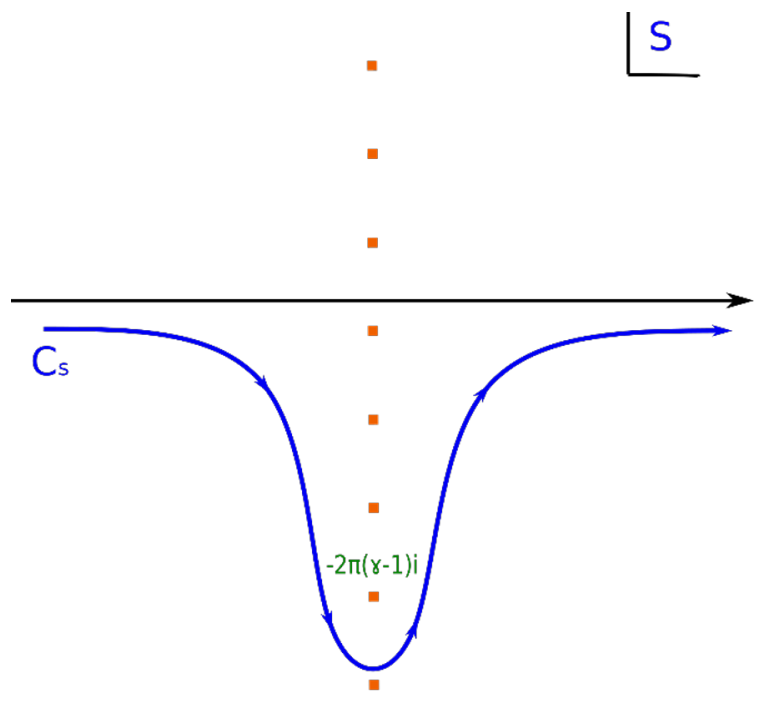

Figure 2. The contour $C_{s}$ of the integral (4.16)(blue line). Orange dots are the poles $s_{2}^{k}=2 \pi i(k-\gamma)$ of the kernel $\mathcal{K}_{\gamma}^{(2)}(s)$.

It is also worth emphasizing that when $-1<\gamma<1$, the contour gets simplified,

$$
\int_{C_{s}} d s \mathcal{K}_{\gamma}^{(2)}(s) G(s)=\int_{-\infty-i \epsilon}^{\infty-i \epsilon} d s \mathcal{K}_{\gamma}^{(2)}(s) G(s)
$$

\subsection{Contour choice: $n \geq 3$ terms}

Now we fix all the contours $C_{k}$ in the integral (4.15).

In the above derivation we have used following formula,

$$
I_{1}(\xi, \beta+i \epsilon)=\int_{-\infty}^{\infty} d \omega \frac{e^{-\omega \xi}}{(\beta+i \epsilon)-e^{-2 \pi \omega}}=\beta^{\left(\frac{\xi}{2 \pi}-1\right)}\left(\frac{e^{i \frac{\xi}{2}}}{2 \sin \frac{\xi}{2}}\right)
$$

Notice that $\xi=p+i t$, and $p$ was a real number. In order for the integral to have an inverse, we need to make sure the choice of the contour $C_{t}$

$$
\int_{C_{t}} d t I(p+i t, \beta) e^{i \omega t}=\frac{e^{-\omega p}}{\beta-e^{-2 \pi \omega}}
$$

The integrand has poles at $s_{n}=i p+2 \pi n$. By an explicit calculation, we recognize that we need pick up poles with $n \geq 1$, thus

$$
\int_{C} d t \equiv \int_{-\infty+i(p+\epsilon)}^{\infty+i(p+\epsilon)} d t
$$


This in particular means that

$$
\begin{aligned}
\mathcal{K}_{n}^{\gamma}\left(\omega_{1}, \cdots \omega_{n}\right) & =\int_{C} \frac{d z}{2 \pi i} z^{\gamma} \frac{e^{2 \pi(\gamma-1) \omega_{1}}}{\left(z-e^{-2 \pi \omega_{1}}\right)^{2}} \prod_{i=2}^{n} \frac{e^{-2 \pi \omega_{i}}}{z-e^{-2 \pi \omega_{i}}} \\
& =\prod_{k=1}^{n} \int_{-\infty+i\left(p_{k}+\epsilon\right)}^{\infty+i\left(p_{k}+\epsilon\right)} d t_{k} e^{i \omega_{k} t_{k}} \int_{C} \frac{d z}{2 \pi i} z^{\gamma} \prod_{k=1}^{n} I\left(i t_{k}+p_{k}, z\right) \\
& =\prod_{k=1}^{n-1} \int_{C_{k}} d s_{k} e^{i \sum_{k=1}^{n-1} s_{k} a_{k}} \mathcal{K}_{n}^{\gamma}\left(s_{1}, \cdots s_{n-1}\right)
\end{aligned}
$$

Therefore we need to choose the following contours,

$$
\operatorname{Im} s_{1}=-2 \pi(\gamma-\epsilon), \quad \operatorname{Im} s_{k}-\operatorname{Im} s_{k-1}=\epsilon, \quad \operatorname{Im} s_{n-1}=-\epsilon
$$

In particular when $\gamma<0$ there is no consistent contour choice for $n \geq 3$ terms.

\section{Applications to conformal field theory}

The discussion so far is quite general, applicable to any density matrices of any theories. From now on, we would like to apply the formula to a special type of reduced density matrices in conformal field theory $(\mathrm{CFT})$. For this purpose, we first briefly summarize the construction of the reduced density matrices. For detailed discussions we refer to [11].

\section{$5.1 \quad$ Set up}

We start from a CFT on $d$ dimensional cylinder $\mathbb{R} \times S^{d-1}$,

$$
d s^{2}=d t^{2}+d \theta^{2}+\sin ^{2} \theta d \Omega_{d-2}^{2} .
$$

We consider a ball shaped subsystem $A$, which is given by

$$
A:\left[0, \theta_{0}\right] \times S^{d-2}, \quad t=0,
$$

and a reduced density matrix $\rho_{V}$ of a globally excited state $|V\rangle$ on the region $A$,

$$
\rho_{V}=\operatorname{tr}_{A^{c}}|V\rangle\langle V| .
$$

The reduced density matrix has a path integral representation on the cylinder with a branch cut on A. The branched cylinder is mapped to $S^{1} \times H^{d-1}$ with the metric [7],

$$
d s^{2}=d \tau^{2}+d u^{2}+\sinh ^{2} u d \Omega_{d-2}^{2}, \quad \tau \sim \tau+2 \pi .
$$

We find that in this frame $\rho_{V}$ has following expression [11],

$$
\rho_{V}=\frac{e^{-\pi K} V\left(\theta_{0}\right) V\left(-\theta_{0}\right) e^{-\pi K}}{\left\langle V\left(\theta_{0}\right) V\left(-\theta_{0}\right)\right\rangle}
$$

where $K$ is the generator of the translation along $\tau$ direction, which can be identified with the modular Hamiltonian of $\rho_{0}$ and $V\left( \pm \theta_{0}\right)$ are local operators corresponding to the 
excited states through state operator correspondence, located at $\tau= \pm \theta_{0}, u=0$. In the small subsystem limit $\theta_{0} \rightarrow 0, V\left(\theta_{0}\right) \rightarrow V\left(-\theta_{0}\right)$.

In this limit we can split the density matrix into the vacuum one $\rho_{0}=e^{-2 \pi K}$ and the rest, $\rho_{V}=\rho_{0}+\delta \rho$. We do so by taking operator product expansion (OPE) of the two local operators,

$$
\rho_{V}=\rho_{0}+e^{-\pi K}\left[\sum_{\mathcal{O}: \text { primaries }} C_{V V}^{\mathcal{O}} B_{\mathcal{O}}\left(\theta_{0},-\theta_{0}\right)\right] e^{-\pi K}
$$

where the index $\mathcal{O}$ labels non identity primaries, and $C_{V V}^{\mathcal{O}}, B_{\mathcal{O}}\left(\theta_{0},-\theta_{0}\right)$ are the $\mathrm{OPE}$ coefficient and the OPE block of $\mathcal{O}$ respectively.

\subsection{The perturbative expression of $T_{\gamma}(\rho)$}

Now we determine the perturbative expression of $T_{\gamma}(\delta \rho)$ in CFT from (4.8). We write,

$$
\operatorname{tr} \rho^{\gamma}=\operatorname{tr} \rho_{0}^{\gamma}+\sum T_{\gamma}^{(n)}(\delta \rho)
$$

and for convenience we reproduce the expression of $T_{\gamma}^{(n)}$ explicitly.

$$
T_{\gamma}^{(n)}(\delta \rho)=\int d s_{1} \cdots d s_{n-1} \mathcal{K}_{\gamma}^{(n)}\left(s_{1}, \cdots s_{n-1}\right) \operatorname{tr}\left[e^{-2 \pi \gamma K} \prod_{k=1}^{n} e^{i K s_{k}} \tilde{\delta} \rho e^{-i K s_{k}}\right] .
$$

Since $\tilde{\delta} \rho=e^{\pi K} \delta \rho e^{\pi K}$, in our case we have

$$
e^{i K s} \delta \tilde{\rho} e^{-i K s}=\sum_{\mathcal{O}: \text { primaries }} C_{V V}^{\mathcal{O}} B_{\mathcal{O}}\left(i s+\theta_{0}, i s-\theta_{0}\right) .
$$

For our $\delta \rho$, the trace in (5.8) can be regarded as a correlation function of the OPE blocks on the covering space $\Sigma_{\gamma}=S_{\gamma}^{1} \times H^{d-1}$, with the metric (5.4) but the periodicity of the Euclidean time direction is changed $\tau \sim \tau+2 \pi \gamma$,

$$
\langle\cdots\rangle_{\Sigma_{\gamma}} \equiv \frac{1}{Z_{\gamma}} \operatorname{tr}\left[e^{-2 \pi \gamma K} \cdots\right],
$$

where $Z_{\gamma}$ is the CFT partition function on this space.

Combining these we can write each term of $T_{\gamma}(\delta \rho)$ by an integral of correlation functions of OPE blocks on $\Sigma_{\gamma}$ along modular flow of vacuum $\rho_{0}$.

$$
\begin{aligned}
\frac{1}{Z_{\gamma}} T_{\gamma}^{(n)}(\delta \rho)= & \sum_{\left\{\mathcal{O}_{l}\right\}} \prod_{l=1}^{n} C_{V V}^{\mathcal{O}_{l}} \int d s_{1} \cdots d s_{n-1} \mathcal{K}_{\gamma}^{(n)}\left(s_{1}, \cdots s_{n-1}\right) \\
& \times\left\langle\prod_{k}^{n-1} B_{\mathcal{O}_{k}}\left(i s_{k}+\theta_{0}, i s_{k}-\theta_{0}\right) B_{\mathcal{O}}\left(\theta_{0},-\theta_{0}\right)\right\rangle_{\Sigma_{\gamma}}
\end{aligned}
$$




\subsection{Bring $n=2$ term to the standard form}

We have seen $n=2$ term is given by

$$
\frac{1}{Z_{\gamma}} T_{\gamma}^{(2)}(\delta \rho)=\sum_{\mathcal{O}: \text { primaries }} \int_{C} d s \mathcal{K}_{\gamma}^{(2)}(s)\left\langle B_{\mathcal{O}}\left(i s+\theta_{0}, i s-\theta_{0}\right) B_{\mathcal{O}}\left(\theta_{0},-\theta_{0}\right)\right\rangle_{\Sigma_{\gamma}}
$$

We can simplify this expression when $0<\gamma<1$, and compare it with known results. In order to do so, let us focus on the contribution $T_{\gamma, \mathcal{O}}^{(2)}(\delta \rho)$ of a particular primary $\mathcal{O}$ to the $n=2$ term. Since the OPE block $B_{\mathcal{O}}$ is summing up descendants of the primary $\mathcal{O}$,we can write it as

$$
B_{\mathcal{O}}\left(\theta_{0},-\theta_{0}\right)=\left.C\left(\theta_{0}, \partial_{a}\right) \mathcal{O}\left(\tau_{a}\right)\right|_{\tau_{a}=0}
$$

where $C\left(\theta_{0}, \partial_{a}\right)$ is a differential operator, and $\tau_{a}$ is the coordinate of Euclidean timelike direction. In the above we did not manifest the dependence of $\mathcal{O}$ on the coordinates of hyperbolic space. The main ingredient of the formula is the integral of two point function,

$$
I_{a b}=\frac{i}{8 \pi^{2}} \int_{-\infty-i \epsilon}^{\infty-i \epsilon} d s \frac{s+2 \pi i \gamma}{\sinh \frac{s}{2} \sinh \frac{s+2 \pi i \gamma}{2}} G_{a b}(s), \quad G_{a b}(s)=\left\langle\mathcal{O}\left(i s+\tau_{a}\right) \mathcal{O}\left(\tau_{b}\right)\right\rangle_{\Sigma_{\gamma}},
$$

and we can write,

$$
T_{\gamma, \mathcal{O}}^{(2)}(\delta \rho)=C\left(\theta_{0}, \partial_{a}\right) C\left(\theta_{0}, \partial_{b}\right) I_{a b}
$$

As we explain in appendix C, we can obtain a simpler expression of $T_{\gamma, \mathcal{O}}^{(2)}(\delta \rho)$,

$$
T_{\gamma, \mathcal{O}}^{(2)}(\delta \rho)=\frac{\gamma \sin \pi \gamma}{4 \pi} C\left(\theta_{0}, \partial_{a}\right) C\left(\theta_{0}, \partial_{b}\right) \int_{-\infty}^{\infty} \frac{d s}{\sinh \frac{s-\pi i \gamma}{2} \sinh \frac{s+\pi i \gamma}{2}} G_{a b}(s-\pi i \gamma)
$$

Notice that in the $\gamma \rightarrow 1$ limit, its derivative recovers the contribution of $\mathcal{O}$ to the second order term $S^{(2)}(\delta \rho)$ of entanglement entropy [8],

$$
S_{\mathcal{O}}^{(2)}(\delta \rho)=C\left(\theta_{0}, \partial_{a}\right) C\left(\theta_{0}, \partial_{b}\right) \int_{-\infty}^{\infty} d s \frac{-1}{4 \sinh ^{2}\left(\frac{s-i \epsilon}{2}\right)}\left\langle\mathcal{O}\left(i s+\tau_{a}\right) \mathcal{O}\left(\tau_{b}\right)\right\rangle_{\Sigma_{1}}
$$

\section{Expansion of Petz's quasi entropy $D_{\gamma}(\rho \| \sigma)$}

In this section, we consider a similar perturbative expansion for Petz's quasi entropy [16], defined by

$$
D_{\gamma}(\rho \| \sigma)=\operatorname{tr} \rho^{\gamma} \sigma^{1-\gamma} .
$$

In this section we consider the case where the one of the reduced density matrices is vacuum $\sigma=\rho_{0}$. We then write $\rho=\rho_{0}+\delta \rho$,

$$
D_{\gamma}\left(\rho \| \rho_{0}\right)=\sum_{n=2}^{\infty} D_{\gamma}^{(n)}(\delta \rho)
$$

The derivation of the perturbative series is very similar to the one of $T_{\gamma}(\rho)$. We first write

$$
D_{\gamma}\left(\rho \| \rho_{0}\right)=\int_{C} \frac{d z}{2 \pi i} z^{\gamma} \operatorname{tr} \frac{\rho_{0}^{1-\gamma}}{z-\rho}
$$


then by expanding the denominator we obtain a similar perturbative series. One notable difference is that in the power series of $D_{\gamma}\left(\rho \| \rho_{0}\right)$, the $\rho_{0}^{\gamma}$ factor appears in the expansion (5.8) is canceled with the $\rho_{0}^{1-\gamma}$ factor which appear in the definition (6.1). The explicit expression of $D_{\gamma}^{(n)}(\delta \rho)$ is given by

$$
\begin{aligned}
D_{\gamma}^{(n)}(\delta \rho)= & \sum_{\left\{\mathcal{O}_{k}\right\}} \prod_{k=1}^{n} C_{V V}^{\mathcal{O}_{k}} \prod_{k=1}^{n-1} d s_{k} \int d s_{k} \mathcal{K}_{\gamma}^{(n)}\left(s_{1}, \cdots s_{n-1}\right) \\
& \times\left\langle\prod_{k}^{n-1} B_{\mathcal{O}_{k}}\left(i s_{k}+\theta_{0}, i s_{k}-\theta_{0}\right) B_{\mathcal{O}_{n}}\left(\theta_{0},-\theta_{0}\right)\right\rangle_{\Sigma_{1}}
\end{aligned}
$$

with the kernel $\mathcal{K}_{\gamma}^{(n)}\left(s_{1}, \cdots s_{n-1}\right)$ defined in (4.14).

One advantage of this quantity is that we can expand it in terms of correlation functions on the space without branch cut, $\Sigma_{1}$, on the contrary to Rényi entropy itself, which is expanded by correlators $\langle\cdots\rangle_{\Sigma_{\gamma}}$ on the space $\Sigma_{\gamma}$ with branch cuts, and they are highly theory dependent quantities. This implies that first few terms of $D_{\gamma}(\rho \| \sigma)$ are theory independent, and allows us to write them holographically.

We also emphasize that the expressions (6.4) are only valid in some range of $\gamma$. In particular higher order terms $D_{\gamma}^{(n)}(\delta \rho), n \geq 3$, has an expression in terms of a modular flow integral only in the range $0<\gamma<1$. The limitation is again coming from the fact that there is a consistent contour choice of the modular flow integrals (4.26) only in the range. However $n=2$ term is still computable by the modular flow integral for any value of $\gamma$.

Below, we will be focusing on following quantity,

$$
Z_{\gamma}(\rho \| \sigma) \equiv D_{-\gamma}(\rho \| \sigma)-D_{\gamma}(\rho \| \sigma)
$$

and its quadratic part,

$$
\left.Y_{\gamma}(\delta \rho) \equiv \frac{d^{2}}{d t^{2}} Z_{\gamma}\left(\sigma+t \delta \rho \| \rho_{0}\right)\right|_{t=0},
$$

as well as its derivative with respect to the index $\gamma$,

$$
X_{\gamma}(\delta \rho)=\frac{d}{d \gamma} Y_{\gamma}(\delta \rho)
$$

Notice that when $\gamma=0 \partial_{\gamma} Z_{\gamma}(\rho \| \sigma)$ reduces to the relative entropy

$$
\left.\partial_{\gamma} Z_{\gamma}(\rho \| \sigma)\right|_{\gamma=0}=2 S(\sigma \| \rho)
$$

in which the order of two density matrices is flipped $\rho \leftrightarrow \sigma$, and $X_{\gamma}(\delta \rho)$ reduces to the Fisher information, which is symmetric under the exchange.

$$
\left.X_{\gamma}(\delta \rho)\right|_{\gamma=0}=F(\rho \| \sigma) .
$$

\subsection{Expressing $X_{\gamma}(\delta \rho)$ and $Y_{\gamma}(\delta \rho)$ by modular flow integrals}

Below we will focus on the range of the Rényi index $-1<\gamma<1$ for $D_{\gamma}^{(2)}(\delta \rho)$, or equivalently $0<\gamma<1$ for $X_{\gamma}(\delta \rho)$ and $Y_{\gamma}(\delta \rho)$. 
When the Rényi index is in the window, $Y_{\gamma}(\delta \rho)$ has following simple modular flow integral representation,

$$
Y_{\gamma}(\delta \rho)=\int_{-\infty-i \epsilon}^{\infty-i \epsilon}\left[\mathcal{K}_{-\gamma}^{(2)}(s)-\mathcal{K}_{\gamma}^{(2)}(s)\right] \operatorname{tr}\left[e^{-2 \pi K} \tilde{\delta} \rho(s) \tilde{\delta} \rho\right] d s
$$

$\mathcal{K}_{\gamma}^{(2)}(s)$ is given by

$$
\mathcal{K}_{\gamma}^{(2)}(s)=\frac{i \sin \pi \gamma}{8 \pi^{2}} \frac{(s+2 \pi i \gamma)}{\sinh \frac{s}{2} \sinh \frac{s+2 \pi i \gamma}{2}}
$$

For the class of $\delta \rho$ we are interested in, we have

$$
\begin{aligned}
Y_{\gamma}(\delta \rho) & =C\left(\theta_{0}, \partial_{a}\right) C\left(\theta_{0}, \partial_{b}\right) \int_{-\infty-i \epsilon}^{\infty-i \epsilon}\left[\mathcal{K}_{-\gamma}^{(2)}(s)-\mathcal{K}_{\gamma}^{(2)}(-s-2 \pi i)\right]\left\langle\mathcal{O}\left(i s+\tau_{a}\right) \mathcal{O}\left(\tau_{b}\right)\right\rangle_{\Sigma_{1}} d s \\
& =C\left(\theta_{0}, \partial_{a}\right) C\left(\theta_{0}, \partial_{b}\right) \int_{-\infty}^{\infty} d s\left[\frac{-(\sin \pi \gamma) / 4 \pi}{\sinh \left(\frac{s-i \epsilon}{2}\right) \sinh \left(\frac{s-2 \pi i \gamma}{2}\right)}\right]\left\langle\mathcal{O}\left(i s+\tau_{a}\right) \mathcal{O}\left(\tau_{b}\right)\right\rangle_{\Sigma_{1}} .
\end{aligned}
$$

In the second term of the first line, we used another expression of $D_{\gamma}^{2}(\delta \rho)$

$$
\begin{aligned}
D_{\gamma}^{2}(\delta \rho) & =C\left(\theta_{0}, \partial_{a}\right) C\left(\theta_{0}, \partial_{b}\right) I_{b a} \\
I_{b a} & =\int_{-\infty+i \epsilon}^{\infty+i \epsilon} d s \mathcal{K}_{\gamma}^{(2)}(s-2 \pi i)\left\langle\mathcal{O}\left(i s+\tau_{b}\right) \mathcal{O}\left(\tau_{a}\right)\right\rangle_{\Sigma_{1}}, \quad \tau_{a}>\tau_{b}
\end{aligned}
$$

and flipped the sign of the integration variable $s \rightarrow-s$. The derivation of this expression is the same with that of (C.12) in appendix C.

By taking derivative of (6.12) with respect to $\gamma$, we have an expression of $X_{\gamma}(\delta \rho)$,

$$
X_{\gamma}(\delta \rho)=C\left(\theta_{0}, \partial_{a}\right) C\left(\theta_{0}, \partial_{b}\right) \int_{-\infty}^{\infty} d s \frac{-1}{4 \sinh ^{2}\left(\frac{s-2 \pi i \gamma}{2}\right)}\left\langle\mathcal{O}\left(i s+\tau_{a}\right) \mathcal{O}\left(\tau_{b}\right)\right\rangle_{\Sigma_{1}} .
$$

\subsection{Holographic expressions of $X_{\gamma}(\delta \rho)$ and $Y_{\gamma}(\delta \rho)$}

So far we have obtained quadratic term $Y_{\gamma}(\delta \rho)$ which is particular linear combination of the Rényi relative divergence $Z_{\gamma}\left(\delta \| \rho_{0}\right)$, and its derivative $X_{\gamma}(\delta \rho)$ in terms of modular flow integral (6.12), (6.14).

As we will see below, through AdS/CFT correspondence, they have simple bulk expressions. The derivations are parallel to the argument of [10], where they obtained the holographic expression of quadratic term of the entanglement entropy $S^{(2)}(\delta \rho)$.

\subsubsection{Set up}

To explain this let us first recall the corresponding bulk set up. Our reference state is the vacuum reduced density matrix $\rho_{0}$, and since we take the subsystem $A$ to be a ball shape 
region, corresponding Ryu Takayanagi surface can be regarded as the bifurcation surface $r_{B}=1$ of the topological black hole,

$$
d s^{2}=-\left(r_{B}^{2}-1\right) d s_{B}^{2}+\frac{d r_{B}^{2}}{\left(r_{B}^{2}-1\right)}+r_{B}^{2} d H_{d-1}^{2}
$$

where $d H_{d-1}^{2}$ denotes the metric of $d-1$ dimensional hyperbolic space,

$$
d H_{d-1}^{2}=d u^{2}+\sinh ^{2} u d \Omega_{d-2}^{2} .
$$

In [10] it was shown that the CFT two point function in (6.12), (6.14) can be written in terms of the bulk symplectic form $\omega_{\phi}$ of the bulk field $\phi$ dual to the CFT primary $\mathcal{O}$,

$$
\left\langle\mathcal{O}\left(i s+\tau_{a}\right) \mathcal{O}\left(\tau_{b}\right)\right\rangle_{\Sigma_{1}}=-\int d X_{B} \omega_{\phi}\left(K_{E}\left(X_{B} \mid \tau_{b a}\right), K_{R}\left(X_{B} \mid s\right)\right) .
$$

We evaluate the integral on fixed $r_{B}=r_{0}$ surface of the topological black hole (6.15), and collectively denote the coordinates of the surface by $X_{B}$. The bulk symplectic form is given by

$$
\omega_{\phi}\left(\delta \phi_{1}, \delta \phi_{2}\right)=n^{M}\left(\delta \phi_{1} \partial_{M} \delta \phi_{2}-\delta \phi_{2} \partial_{M} \delta \phi_{1}\right)
$$

where $n^{M}$ is the normal vector of the $r_{B}=r_{0}$ surface. $K_{E}\left(X_{B} \mid \tau_{b a}\right), K_{R}\left(X_{B} \mid s\right)$ are the Euclidean and Retarded bulk to boundary propagator of the bulk field $\phi$, respectively. The primary operators in the CFT two point function are located at the origin of the hyperbolic space $u=0$. We omit this information in the bulk to boundary propagators.

\subsubsection{Holographic rewritings}

By plugging (6.17) into (6.12), and evaluating the remaining $s$ integral by picking up poles of the kernel, we get ${ }^{1}$

$$
Y_{\gamma}(\delta \rho)=i C\left(\theta_{0}, \partial_{a}\right) C\left(\theta_{0}, \partial_{b}\right) \int d X_{B} \omega_{\phi}\left(K_{E}\left(X_{B} \mid \tau_{b a}\right), K_{E}\left(X_{B} \mid-2 \pi \gamma\right)-K_{E}\left(X_{B} \mid 0\right)\right)
$$

By shifting the time coordinate $s_{B} \rightarrow s_{B}+i \tau_{a}$, and using the relation between the Euclidean bulk to boundary propagator and the expectation value of the bulk scalar field operator $\phi\left(X_{B}\right)$,

$$
C\left(\theta_{0}, \partial_{a}\right) K_{E}\left(X_{B} \mid \tau_{a}\right)=\left\langle V\left|\phi\left(X_{B}\right)\right| V\right\rangle \equiv\left\langle\phi\left(X_{B}\right)\right\rangle_{V}
$$

we get,

$$
Y_{\gamma}(\delta \rho)=i \int d X_{B} \omega_{\phi}\left(\langle\phi(0)\rangle_{V},\langle\phi(2 \pi \gamma)\rangle_{V}-\langle\phi(0)\rangle_{V}\right),
$$

where $\langle\phi(2 \pi \gamma)\rangle_{V}$ is the expectation value of the bulk field rotated by $2 \pi \gamma$ along the Euclidean timelike direction,

$$
\langle\phi(2 \pi \gamma)\rangle_{V} \equiv \operatorname{tr}\left[\rho_{V} e^{-2 \pi \gamma K} \phi e^{2 \pi \gamma K}\right]
$$

\footnotetext{
${ }^{1}$ The argument here is very similar to the one in [10]. See appendix E for the details.
} 
In the argument of the bulk local field $\phi$, we only manifested the Euclidean time like coordinate,

$$
\phi(\tau) \equiv \phi\left(r_{B}, \tau+i s_{B}, u, \Omega_{d-2}\right) .
$$

We can obtain a similar expression for $X_{\gamma}(\delta \rho)$ just by taking a derivative of $Y_{\gamma}(\delta \rho)$,

$$
X_{\gamma}(\delta \rho)=-2 \pi \int d X_{B} \omega_{\phi}\left(\langle\phi(0)\rangle_{V}, \partial_{s}\langle\phi(2 \pi \gamma)\rangle_{V}\right)
$$

here we used the relation $\partial_{\gamma}=-i \partial_{s}$. This integral is invariant under the deformation of the surface on which we are evaluating the integral. In particular we can choose the fixed time slice $s_{B}=0$, then the integral can be written as,

$$
X_{\gamma}(\delta \rho)=-2 \pi \int_{\Sigma} d \Sigma^{a} \xi^{b} T_{a b}\left(\langle\phi(0)\rangle_{V},\langle\phi(2 \pi \gamma)\rangle_{V}\right)
$$

where $\Sigma$ is the bulk region on the time slice $s_{B}=0$, which is enclosed by the boundary subsystem A and the bifurcation surface of the topological black hole (ie, RT surface). Also $d \Sigma^{a}$ is the volume element of $\Sigma$, and $\xi^{b}$ is the timelike Killing vector of the black hole. $T_{a b}$ is a quadratic form of $\phi$ related to the stress energy tensor of the bulk field,

$$
T_{a b}\left(\phi_{1}, \phi_{2}\right)=\partial_{a} \phi_{1} \partial_{b} \phi_{2}-m^{2} g_{a b} \phi_{1} \phi_{2}
$$

There is another way to derive this result. Let us come back to the CFT formula,

$$
X_{\gamma}(\delta \rho)=C\left(\theta_{0}, \partial_{a}\right) C\left(\theta_{0}, \partial_{b}\right) \int_{-\infty}^{\infty} d s \frac{-1}{4 \sinh ^{2}\left(\frac{s-2 \pi i \gamma}{2}\right)}\left\langle\mathcal{O}\left(i s+\tau_{a}\right) \mathcal{O}\left(\tau_{b}\right)\right\rangle_{\Sigma_{1}}
$$

by changing the integration variable to $t=s-2 \pi i \gamma$ and shifting the contour we get,

$$
\begin{aligned}
X_{\gamma}(\delta \rho) & =C\left(\theta_{0}, \partial_{a}\right) C\left(\theta_{0}, \partial_{b}\right) \int_{-\infty}^{\infty} d t \frac{-1}{4 \sinh ^{2}\left(\frac{t-2 \pi i \epsilon}{2}\right)}\left\langle\mathcal{O}\left(i(t+2 \pi i \gamma)+\tau_{a}\right) \mathcal{O}\left(\tau_{b}\right)\right\rangle_{\Sigma_{1}} \\
& =\int_{-\infty}^{\infty} d s \frac{-1}{4 \sinh ^{2}\left(\frac{s-2 \pi i \epsilon}{2}\right)} \operatorname{tr}\left[\tilde{\delta} \rho(s) e^{2 \pi \gamma} \tilde{\delta} \rho e^{-2 \pi \gamma}\right]
\end{aligned}
$$

In [11] it was shown that the excited state modular Hamiltonian $K_{\rho}$ of $\rho$, when expanded by $\delta \rho$, the leading order correction to the vacuum modular Hamiltonian $K$ is given by

$$
K_{\rho}=K+\int_{-\infty}^{\infty} \frac{d s}{\sinh ^{2} \frac{s}{2}} \tilde{\delta} \rho(s) \equiv K+\delta K
$$

It was also shown that contribution of a primary operator $\mathcal{O}$ to the correction $\delta K$ has a bulk expression

$$
\delta K=2 \pi \int_{\Sigma} d \Sigma^{a} \xi^{b} T_{a b}\left(\langle\phi(0)\rangle_{V}, \hat{\phi}\right)
$$

where $\hat{\phi}$ is the bulk field operator dual to $\mathcal{O}$. By plugging this into (6.28), we recover the result. 


\section{Conclusions}

In this paper we developed a novel way to perturbatively expand Rényi tpe quantities involving powers of reduced density matrices. We then obtained a holographic expression of the quadratic parts of Rényi relative divergences $X_{\gamma}(\delta \rho), Y_{\gamma}(\delta \rho)$ in terms of bulk symplectic form starting from the CFT calculations.

It is interesting find a bulk derivation of this result. One difficulty in doing so is coming form the fact that in general there is no nice path integral representation of Rényi relative divergence. This is because even if reduced density matrices $\rho, \sigma$ can be written by path integrals, $\rho^{\gamma}$ and $\sigma^{1-\gamma}$ can not. If we could find such a representation, then we can map the CFT path integral calculationss to the bulk on shell action calculations. Indeed, in a special case where Rényi relative divergence can be represented by a path integral, corresponding holographic calcuation is known [21]. However in order to derive a bulk formula for Rényi relative divergence between two generic bulk configurations, we need to take a different approach. A possible approach would be first going back to replica trick [32], compute $\operatorname{tr} \rho^{n} \sigma^{m}$ for positive integers $n, m$ then analytically continue the result $n \rightarrow \gamma, m \rightarrow 1-\gamma$.

Furthermore it would be nice if we could read off finer information of bulk geometries using Rényi relative divergence. It has been shown that using relative entropy, we can read off first non linear part of Einstein equations $[8,10]$ in particular. Since Rényi relative divergence is a one parameter generalization of relative entropy, and knows about details of eigenvalue distribution of excited state reduced density matrices, it is natural to expect this.

Another interesting direction would be to calculate correlation functions with insertions of modular flows of excited states, by using the technique developed in this paper. For example [33-35], two point function with an insertion of a modular flow $\left\langle\mathcal{O}(x) \Delta^{i t} \mathcal{O}(y)\right\rangle$ was considered. There, it was also argued that this is useful to extract information of corresponding bulk geometry. Naively speaking we can perturbatively compute them by Wick rotating the Rényi index $\gamma$ to the imaginary value $\gamma \rightarrow i t$ in our result. The task would be to check that there is no obstacle to do this.

\section{Acknowledgments}

We thank Alex Belin, Tom Faulkner, Sudip Ghosh, Norihiro Iizuka, Robert Myers,Tatsuma Nishioka, Jonathan Oppenheim, Gábor Sárosi, Tadashi Takayanagi and Kotaro Tamaoka for discussions.

\section{A The calculation of $\mathcal{K}_{\gamma}^{(n)}\left(s_{1}, \cdots s_{n-1}\right)$}

In this appendix, we explain the details of the calculation of the kernel $\mathcal{K}_{\gamma}^{(n)}\left(s_{1}, \cdots s_{n-1}\right)$, starting from (4.12).

In order to do this, we first decompose $J(z)$ in (4.12)

$$
J(z)=z^{\gamma} I_{2}\left(\xi_{1}, z\right) \prod_{k=2}^{n-1} I_{1}\left(\xi_{k}, z\right) I_{1}\left(\xi_{n}, z\right),
$$


where

$$
\begin{array}{ll}
\xi_{1}=-2 \pi(\gamma-1)+i\left(s_{1}+q\right), & \xi_{n}=2 \pi-\left(s_{n-1}-q\right) i, \\
\xi_{k}=2 \pi+\left(s_{k}-s_{k-1}+q\right) i, & 2 \leq k \leq n-1,
\end{array}
$$

and

$$
I_{1}(\xi, z)=\int_{-\infty}^{\infty} d \omega \frac{e^{-\omega \xi}}{z-e^{-2 \pi \omega}}, \quad I_{2}(\xi, z)=\int_{-\infty}^{\infty} d \omega \frac{e^{-\omega \xi}}{\left(z-e^{-2 \pi \omega}\right)^{2}}
$$

For $I_{1}(\xi, z)$, by carefully picking up the contributions of the relevant poles we have,

$$
I_{1}(\xi, \beta+i \epsilon)=\beta^{\left(\frac{\xi}{2 \pi}-1\right)}\left(\frac{e^{-i \frac{\xi}{2}}}{2 \sin \frac{\xi}{2}}\right), \quad I_{1}(\xi, \beta-i \epsilon)=\beta^{\left(\frac{\xi}{2 \pi}-1\right)}\left(\frac{e^{i \frac{\xi}{2}}}{2 \sin \frac{\xi}{2}}\right) .
$$

One way to check these is using

$$
\frac{1}{z+i \epsilon}-\frac{1}{z-i \epsilon}=-2 \pi i \delta(z)
$$

Then,

$$
\begin{aligned}
\operatorname{Disc} I & =\lim _{\epsilon \rightarrow 0_{+}}[I(z+i \epsilon)-I(z-i \epsilon)] \\
& =-2 \pi i \int_{-\infty}^{\infty} d \omega e^{-\xi \omega} \delta\left(\beta-e^{-2 \pi \omega}\right)=-i \beta\left(\frac{\xi}{2 \pi}-1\right)
\end{aligned}
$$

This is consistent with (A.5).

We can evaluate $I_{2}(\xi, z)$ just by taking derivative of $I_{1}(\xi, z)$ with respect to $\beta$,

$$
\begin{aligned}
& I_{2}(\xi, \beta+i \epsilon)=-\left(\frac{\xi}{2 \pi}-1\right) \beta^{\left(\frac{\xi}{2 \pi}-2\right)}\left(\frac{e^{-i \frac{\xi}{2}}}{2 \sin \frac{\xi}{2}}\right), \\
& I_{2}(\xi, \beta-i \epsilon)=-\left(\frac{\xi}{2 \pi}-1\right) \beta^{\left(\frac{\xi}{2 \pi}-2\right)}\left(\frac{e^{i \frac{\xi}{2}}}{2 \sin \frac{\xi}{2}}\right) .
\end{aligned}
$$

Combining these, we obtain the relevant expressions of $J(z)$

$$
J(\beta+i \epsilon)=-\beta^{\left(\gamma+\sum_{k=1}^{n} \frac{\xi_{k}}{2 \pi}-(n+1)\right)} \frac{\left(\frac{\xi_{1}}{2 \pi}-1\right)}{\prod_{k=1}^{n} 2 \sin \frac{\xi_{k}}{2}} e^{-\frac{i}{2} \sum_{k=1}^{n} \xi_{k}}
$$

and

$$
J(\beta-i \epsilon)=-\beta^{\left(\gamma+\sum_{k=1}^{n} \frac{\xi_{k}}{2 \pi}-(n+1)\right)} \frac{\left(\frac{\xi_{1}}{2 \pi}-1\right)}{\prod_{k=1}^{n} 2 \sin \frac{\xi_{k}}{2}} e^{\frac{i}{2} \sum_{k=1}^{n} \xi_{k}}
$$

Since

$$
\gamma+\sum_{k=1}^{n} \frac{\xi_{k}}{2 \pi}-(n+1)=-1+\frac{i q n}{2 \pi}
$$


the $\beta$ integral produces the delta function,

$$
\int_{-\infty}^{\infty} \frac{d \beta}{2 \pi i} \beta^{-1+\frac{i q n}{2 \pi}}=\frac{2 \pi}{n i} \delta(q) .
$$

By picking up the discontinuity across the real line, we get

$$
\begin{aligned}
\delta(q) \mathcal{K}_{\gamma}^{(n)}\left(s_{1}, \cdots s_{n-1}\right) & =\frac{n}{(2 \pi)^{n}} \int_{0}^{\infty} \frac{d \beta}{2 \pi i}(J(\beta-i \epsilon)-J(\beta+i \epsilon)), \quad \epsilon \rightarrow 0_{+}, \\
& =-\frac{n}{(2 \pi)^{n}}\left(\frac{2 \pi}{n i} \delta(q)\right) \frac{\left(\frac{\xi_{1}}{2 \pi}-1\right)}{\prod_{k=1}^{n} 2 \sin \frac{\xi_{k}}{2}}\left(e^{\frac{i}{2} \sum_{k=1}^{n} \xi_{k}}-e^{-\frac{i}{2} \sum_{k=1}^{n} \xi_{k}}\right)
\end{aligned}
$$

Notice that

$$
\begin{aligned}
e^{-\frac{i}{2} \sum_{k=1}^{n} \xi_{k}}-e^{+\frac{i}{2} \sum_{k=1}^{n} \xi_{k}} & =e^{i \pi(\gamma-n)}-e^{-i \pi(\gamma-n)} \\
& =2 i(-1)^{n} \sin \pi \gamma
\end{aligned}
$$

and

$$
\frac{\left(\frac{\xi_{1}}{2 \pi}-1\right)}{\prod_{k=1}^{n} \sin \frac{\xi_{k}}{2}}=\frac{-i^{n+1}}{2 \pi} \frac{\left(s_{1}+2 \pi i \gamma\right)}{\sinh \left(\frac{s_{1}+2 \pi i \gamma}{2}\right) \prod_{k=2}^{n-1} \sinh \left(\frac{s_{k}-s_{k-1}}{2}\right) \sinh \left(\frac{s_{n-1}}{2}\right)}
$$

From this we finally arrive at the expression of the kernel,

$$
\mathcal{K}_{\gamma}^{(n)}\left(s_{1}, \cdots s_{n-1}\right)=\frac{i}{8 \pi^{2}}\left(\frac{-i}{4 \pi}\right)^{n-2} \frac{\left(s_{1}+2 \pi i \gamma\right) \sin \pi \gamma}{\sinh \left(\frac{s_{1}+2 \pi i \gamma}{2}\right) \prod_{k=2}^{n-1} \sinh \left(\frac{s_{k}-s_{k-1}}{2}\right) \sinh \left(\frac{s_{n-1}}{2}\right)}
$$

\section{B Fixing the contour of $n=2$ term}

In this appendix, we fix the correct contour $C_{s}$ of $n=2$ real time integral

$$
\int_{C_{s}} d s \mathcal{K}_{\gamma}^{(2)}(s) e^{i a s}=\frac{i \sin \pi \gamma}{8 \pi^{2}} \int_{C_{s}} d s \frac{s+2 \pi i \gamma}{\sinh \frac{s}{2} \sinh \frac{s+2 \pi i \gamma}{2}} e^{i a s},
$$

which reproduces the kernel in the frequency representation, (3.3)

$$
\begin{aligned}
\mathcal{K}_{\gamma}^{(2)}\left(\omega_{1}, \omega_{2}\right) & =e^{2 \pi \gamma \omega_{1}} e^{-2 \pi \omega_{1}-2 \pi \omega_{2}} K\left(\omega_{1}, \omega_{2}\right) \\
& =\frac{e^{2 \pi \gamma \omega_{1}} e^{-2 \pi \omega_{1}-2 \pi \omega_{2}}}{\left(e^{-2 \pi \omega_{1}}-e^{-2 \pi \omega_{2}}\right)^{2}}\left[(\gamma-1) e^{-2 \pi \gamma \omega_{1}}+e^{-2 \pi \gamma \omega_{2}}-\gamma e^{-2 \pi(\gamma-1) \omega_{1}} e^{-2 \pi \omega_{2}}\right] .
\end{aligned}
$$

Using $a \equiv \omega_{1}-\omega_{2}$, we have,

$$
\mathcal{K}_{\gamma}^{(2)}(a)=\frac{e^{2 \pi a}}{\left(1-e^{2 \pi a}\right)^{2}}\left[(\gamma-1)+e^{2 \pi a \gamma}-\gamma e^{2 \pi a}\right] .
$$

Let's do the integral (B.1). There are two types of poles.

$$
s_{1}^{n}=2 \pi i n, \quad s_{2}^{k}=2 \pi i(k-\gamma) .
$$


We choose a contour which contains $s_{1}^{n}, n \geq 0$, and $s_{2}^{k}, k \geq 1$. One way to manifest the contour prescription is introducing an additional parameter $x>0$,

$$
\mathcal{K}_{\gamma}^{(2)}(s, x)=\left(\frac{i \sin \pi \gamma}{8 \pi^{2}}\right) \frac{s+2 \pi i \gamma}{\sinh \frac{s+x}{2} \sinh \frac{s+2 \pi i \gamma}{2}},
$$

and finally send $x \rightarrow 0$ to get the desired result.

We have

$$
\operatorname{Res}\left[s_{n}^{1}\right]=\frac{i(n+\gamma)}{2 \pi} e^{-2 \pi a n}, \quad \operatorname{Res}\left[s_{n}^{2}\right]=-\frac{i k}{2 \pi} e^{2 \pi a(\gamma-k)} .
$$

By combining them,

$$
\begin{aligned}
\int_{C_{s}} d s \mathcal{K}_{\gamma}^{(2)}(s) e^{i a s} & =2 \pi i\left(\sum_{n} \operatorname{Res}\left[s_{n}^{1}\right]+\sum_{k} \operatorname{Res}\left[s_{k}^{2}\right]\right) \\
& =-\left[\left(1-e^{-2 \pi a \gamma}\right) \sum_{k} k e^{-2 \pi a k}+\gamma \sum_{n} e^{-2 \pi a \gamma n}\right] \\
& \left.=-\frac{e^{2 \pi a}}{\left(e^{2 \pi a}-1\right)^{2}}(1-\gamma)+\gamma e^{2 \pi a}-e^{2 \pi a \gamma}\right] \\
& =\mathcal{K}_{\gamma}^{(2)}(a) .
\end{aligned}
$$

This is what we want. In the sum, we included $n=0$ contribution.

\section{Simplifying $T_{\gamma}^{(2)}(\delta \rho)$}

In this section we simplify $n=2$ term of $T_{\gamma}^{(2)}(\delta \rho)$. In section 5.3 we saw that the contribution of particular primary $\mathcal{O}$ to $T_{\gamma}^{(2)}(\delta \rho)$ can be written

$$
T_{\gamma, \mathcal{O}}^{(2)}(\delta \rho)=C\left(\theta_{0}, \partial_{a}\right) C\left(\theta_{0}, \partial_{b}\right) I_{a b}
$$

where

$$
I_{a b}=\frac{i}{8 \pi^{2}} \int_{-\infty-i \epsilon}^{\infty-i \epsilon} d s \frac{s+2 \pi i \gamma}{\sinh \frac{s}{2} \sinh \frac{s+2 \pi i \gamma}{2}} G_{a b}(s), \quad G_{a b}(s)=\left\langle\mathcal{O}\left(i s+\tau_{a}\right) \mathcal{O}\left(\tau_{b}\right)\right\rangle_{\Sigma_{\gamma}},
$$

and $C\left(\theta_{0}, \partial_{a}\right)$ is a differential operator summing up all descendants.

This expression only holds when $\tau_{a}>\tau_{b}$. This is because we started from the spectral representation,

$$
I_{a b}=\int d \omega_{1} d \omega_{2} \mathcal{K}^{\gamma}(a) e^{-2 \pi \gamma \omega_{1}}\left\langle\omega_{1}\left|\mathcal{O}\left(\tau_{a}\right)\right| \omega_{2}\right\rangle\left\langle\omega_{2}\left|\mathcal{O}\left(\tau_{b}\right)\right| \omega_{1}\right\rangle,
$$

rewrote it in terms of the modular flow integral by

$$
\mathcal{K}^{\gamma}(a)=\int_{-\infty-i \epsilon}^{\infty-i \epsilon} \mathcal{K}^{\gamma}(s) e^{i a s}, \quad a=\omega_{1}-\omega_{2}
$$


and then undoing the spectral decomposition of the two point function $G_{a b}(s)$,

$$
\int d \omega_{1} d \omega_{2} e^{-2 \pi \gamma \omega_{1}+i a s}\left\langle\omega_{1}\left|\mathcal{O}\left(\tau_{a}\right)\right| \omega_{2}\right\rangle\left\langle\omega_{2}\left|\mathcal{O}\left(\tau_{b}\right)\right| \omega_{1}\right\rangle=\left\langle\mathcal{O}\left(i s+\tau_{a}\right) \mathcal{O}\left(\tau_{b}\right)\right\rangle_{\Sigma_{\gamma}}
$$

The spectral integral only converges when $\tau_{a}>\tau_{b}$.

When $\tau_{b}>\tau_{a}$, we instead write

$$
\begin{aligned}
I_{a b} & =\int d \omega_{1} d \omega_{2}\left[\mathcal{K}^{\gamma}(a) e^{-2 \pi \gamma a}\right] e^{-2 \pi \gamma \omega_{2}}\left\langle\omega_{1}\left|\mathcal{O}\left(\tau_{a}\right)\right| \omega_{2}\right\rangle\left\langle\omega_{2}\left|\mathcal{O}\left(\tau_{b}\right)\right| \omega_{1}\right\rangle, \\
\mathcal{K}^{\gamma}(a) e^{-2 \pi \gamma a} & =\int_{-\infty-i \epsilon}^{\infty-i \epsilon} d s \mathcal{K}^{\gamma}(s) e^{i a(s+2 \pi i \gamma)} \\
& =\int_{-\infty+2 \pi i(\gamma-\epsilon)}^{\infty+2 \pi i(\gamma-\epsilon)} d t \mathcal{K}^{\gamma}(t-2 \pi i \gamma) e^{i a t} .
\end{aligned}
$$

Since

$$
\mathcal{K}^{\gamma}(t-2 \pi i \gamma)=\frac{i \sin \pi \gamma}{8 \pi^{2}} \frac{t}{\sinh \frac{t}{2} \sinh \frac{t-2 \pi i \gamma}{2}}
$$

is regular on the strip $2 \pi(\gamma-\epsilon)>\operatorname{Im} t>0$ when $0<\gamma<1$, we deform the contour to $\operatorname{Im} t=\epsilon$

$$
\mathcal{K}^{\gamma}(a) e^{-2 \pi \gamma a}=\int_{-\infty+i \epsilon}^{\infty+i \epsilon} d t \mathcal{K}^{\gamma}(t-2 \pi i \gamma) e^{i a t}
$$

Therefore for $\tau_{b}>\tau_{a}$ we have

$$
I_{a b}=\int_{-\infty+i \epsilon}^{\infty+i \epsilon} \mathcal{K}^{\gamma}(s-2 \pi i \gamma)\left\langle\mathcal{O}\left(\tau_{b}\right) \mathcal{O}\left(\tau_{a}+i s\right)\right\rangle_{\Sigma_{\gamma}}, \quad \tau_{b}>\tau_{a} .
$$

We have similar formule for $I_{b a}$, just by flipping $\tau_{a} \leftrightarrow \tau_{b}$.

Finally we combine these expressions to get a simpler form of $T_{\gamma}^{(2)}(\delta \rho)$. The two point function in (C.2) is analytic in the strip region $-2 \pi \gamma<\operatorname{Im} s<\tau_{b a}$. Since when $0<\gamma<1$ there is no pole coming from the kernel in the strip, and we are allowed to deform the contour $s \rightarrow s-\pi i \gamma$. Then the integral for $\tau_{a}>\tau_{b}$ becomes

$$
I_{a b}=\frac{i \sin \pi \gamma}{8 \pi^{2}} \int_{-\infty-i \epsilon}^{\infty-i \epsilon} d s \frac{s+\pi i \gamma}{\sinh \frac{s-\pi i \gamma}{2} \sinh \frac{s+\pi i \gamma}{2}} G_{a b}(s-\pi i \gamma), \quad \tau_{a}>\tau_{b}
$$

Now we do a similar thing for $I_{b a}$,

$$
I_{b a}=\int_{-\infty+i \epsilon}^{\infty+i \epsilon} \mathcal{K}^{\gamma}(s-2 \pi i \gamma)\left\langle\mathcal{O}\left(\tau_{a}\right) \mathcal{O}\left(\tau_{b}+i s\right)\right\rangle_{\Sigma_{\gamma}}, \quad \tau_{a}>\tau_{b}
$$

By shifting the contour $s \rightarrow s+\pi i \gamma$, and then flipping the sign $s \rightarrow-s$ we get

$$
I_{b a}=\frac{i \sin \pi \gamma}{8 \pi^{2}} \int_{-\infty+i \epsilon}^{\infty+i \epsilon} d s \frac{-s+\pi i \gamma}{\sinh \frac{s-\pi i \gamma}{2} \sinh \frac{s+\pi i \gamma}{2}} G_{a b}(s-\pi i \gamma)
$$

In the expressions (C.11) (C.13), we can take $\epsilon \rightarrow 0$. Finally we obtain

$$
I_{a b}+I_{b a}=\frac{\gamma \sin \pi \gamma}{4 \pi} \int_{-\infty}^{\infty} \frac{d s}{\sinh \frac{s-\pi i \gamma}{2} \sinh \frac{s+\pi i \gamma}{2}} G_{a b}(s-\pi i \gamma)
$$


$T_{\gamma, \mathcal{O}}^{(2)}(\delta \rho)$ is obtained by applying the differential operator,

$$
T_{\gamma, \mathcal{O}}^{(2)}(\delta \rho)=C\left(\theta_{0}, \partial_{a}\right) C\left(\theta_{0}, \partial_{b}\right)\left(I_{a b}+I_{b a}\right)
$$

Notice that in the $\gamma \rightarrow 1$ limit, its derivative recovers the second order term $S^{(2)}(\delta \rho)$ entanglement entropy,

$$
S_{\mathcal{O}}^{(2)}(\delta \rho)=C\left(\theta_{0}, \partial_{a}\right) C\left(\theta_{0}, \partial_{b}\right) \int_{-\infty}^{\infty} d s \frac{-1}{4 \sinh ^{2}\left(\frac{s-i \epsilon}{2}\right)}\left\langle\mathcal{O}\left(i s+\tau_{a}\right) \mathcal{O}\left(\tau_{b}\right)\right\rangle_{\Sigma_{1}}
$$

\section{Direct Fourier transformation}

Here we would like to directly show that

$$
\begin{aligned}
\mathcal{K}_{n}^{\gamma}(s) & =\int_{-\infty+i \epsilon}^{\infty+i \epsilon} \frac{d a}{2 \pi} \mathcal{K}_{n}^{\gamma}(\omega) e^{-i a s} \\
& =\int_{-\infty+i \epsilon}^{\infty+i \epsilon} \frac{d a}{2 \pi} \frac{e^{-i a s}}{\sinh ^{2} \pi a}\left[(\gamma-1)-\gamma e^{2 \pi a}+e^{2 \pi \gamma a}\right]
\end{aligned}
$$

The first piece is

$$
I_{1}=\int_{-\infty+i \epsilon}^{\infty+i \epsilon} \frac{d a}{2 \pi} \frac{e^{-i a s}}{\sinh ^{2} \pi a}=\frac{s}{4 \pi^{2}}\left(\frac{1}{1-e^{-s}}\right)
$$

The second order term can be obtained by the shift $s \rightarrow s+2 \pi i$, therefore

$$
I_{2}=\int_{-\infty+i \epsilon}^{\infty+i \epsilon} \frac{d a}{2 \pi} \frac{e^{-i a(s+2 \pi i)}}{\sinh ^{2} \pi a}=\frac{(s+2 \pi i)}{4 \pi^{2}}\left(\frac{1}{1-e^{-s}}\right)
$$

Similarly,

$$
I_{3}=\int_{-\infty+i \epsilon}^{\infty+i \epsilon} \frac{d a}{2 \pi} \frac{e^{-i a(s+2 \pi i \gamma)}}{\sinh ^{2} \pi a}=\frac{(s+2 \pi i \gamma)}{4 \pi^{2}}\left(\frac{1}{1-e^{-(s+2 \pi i \gamma)}}\right)
$$

Then the total integral is

$$
(\gamma-1) I_{1}+\gamma I_{2}+I_{3}=\frac{i}{8 \pi^{2}}\left[\frac{(s+2 \pi i \gamma) \sin \pi \gamma}{\sinh \frac{s}{2} \sinh \frac{s+2 \pi i \gamma}{2}}\right]
$$

therefore we recover the first non trivial part.

\section{E Details of the holographic rewriting}

In section 6.2.2, we used the result,

$$
\begin{aligned}
Y_{\gamma}(\delta \rho) & =\int d X_{B} \omega_{\phi}\left(K_{E}\left(X_{B} \mid \tau_{a b}, \int_{-\infty}^{\infty} d s \mathcal{Y}(s-i \epsilon) K_{R}\left(X_{B} \mid s\right)\right)\right. \\
& =i \int d X_{B} \omega_{\phi}\left(K_{E}\left(X_{, B} \mid \tau_{b a}\right), K_{E}\left(X_{B} \mid-2 \pi \gamma\right)-K_{E}\left(X_{B} \mid 0\right)\right)
\end{aligned}
$$


with

$$
\mathcal{Y}(s-i \epsilon)=\frac{-(\sin \pi \gamma) / 4 \pi}{\sinh \left(\frac{s-2 i \epsilon}{2}\right) \sinh \left(\frac{s-2 \pi i \gamma}{2}\right)} .
$$

In this appendix, we prove this. The derivation is very similar to the one in [10].

The retarded bulk to boundary propagator is given by

$$
K_{R}\left(X_{B} \mid s\right)=i \theta\left(s_{B}-s\right) \lim _{\epsilon \rightarrow 0}\left[K_{E}\left(X_{B} \mid i s-\epsilon\right)-K_{E}\left(X_{B} \mid i s+\epsilon\right)\right] .
$$

In particular, as a function of $s$, the retarded propagator is non vanishing only in the window $-\infty<s<s_{*}$. The value of $s_{*}$ is fixed by demanding that the boundary point is null separated from the bulk point $X_{B}$. Then

$$
\begin{aligned}
\int_{-\infty}^{\infty} d s \mathcal{Y}(s-i \epsilon) K_{R}\left(X_{B} \mid s\right) & =\int_{-\infty}^{s_{*}} \mathcal{Y}(i s-\epsilon)\left[K_{E}\left(X_{B} \mid s+i \epsilon\right)-K_{E}\left(X_{B} \mid i s+\epsilon\right)\right] \\
& =\int_{C} d s \mathcal{Y}(s-i \epsilon) K_{E}\left(X_{B} \mid s\right),
\end{aligned}
$$

where $C$ is the closed contour starting from $-\infty+i \epsilon$ to $s_{*}+i \epsilon$, then to $s_{*}+2(\pi-\epsilon) i$ and ending at $-\infty+2(\pi-\epsilon) i$. We also used the KMS condition $K_{E}\left(X_{B} \mid i s+2 \pi\right)=K_{E}\left(X_{B} \mid i s\right)$, $\mathcal{Y}(s+2 \pi i)=\mathcal{Y}(s)$ to fix the contour. By picking up poles of $\mathcal{Y}(s-i \epsilon)$ at $s=i \epsilon$ and $s=2 \pi i \gamma$, we obtain the result.

Open Access. This article is distributed under the terms of the Creative Commons Attribution License (CC-BY 4.0), which permits any use, distribution and reproduction in any medium, provided the original author(s) and source are credited.

\section{References}

[1] S. Ryu and T. Takayanagi, Holographic derivation of entanglement entropy from AdS/CFT, Phys. Rev. Lett. 96 (2006) 181602 [hep-th/0603001] [INSPIRE].

[2] S. Ryu and T. Takayanagi, Aspects of Holographic Entanglement Entropy, JHEP 08 (2006) 045 [hep-th/0605073] [INSPIRE].

[3] V.E. Hubeny, M. Rangamani and T. Takayanagi, A covariant holographic entanglement entropy proposal, JHEP 07 (2007) 062 [arXiv:0705.0016] [INSPIRE].

[4] J. Bhattacharya, M. Nozaki, T. Takayanagi and T. Ugajin, Thermodynamical Property of Entanglement Entropy for Excited States, Phys. Rev. Lett. 110 (2013) 091602 [arXiv: 1212.1164] [INSPIRE].

[5] N. Lashkari, M.B. McDermott and M. Van Raamsdonk, Gravitational dynamics from entanglement 'thermodynamics', JHEP 04 (2014) 195 [arXiv:1308.3716] [INSPIRE].

[6] T. Faulkner, M. Guica, T. Hartman, R.C. Myers and M. Van Raamsdonk, Gravitation from Entanglement in Holographic CFTs, JHEP 03 (2014) 051 [arXiv: 1312.7856] [INSPIRE].

[7] H. Casini, M. Huerta and R.C. Myers, Towards a derivation of holographic entanglement entropy, JHEP 05 (2011) 036 [arXiv: 1102.0440] [INSPIRE].

[8] T. Faulkner, Bulk Emergence and the RG Flow of Entanglement Entropy, JHEP 05 (2015) 033 [arXiv: 1412.5648] [INSPIRE]. 
[9] T. Faulkner, R.G. Leigh and O. Parrikar, Shape Dependence of Entanglement Entropy in Conformal Field Theories, JHEP 04 (2016) 088 [arXiv: 1511.05179] [INSPIRE].

[10] T. Faulkner, F.M. Haehl, E. Hijano, O. Parrikar, C. Rabideau and M. Van Raamsdonk, Nonlinear Gravity from Entanglement in Conformal Field Theories, JHEP 08 (2017) 057 [arXiv: 1705.03026] [INSPIRE].

[11] G. Sárosi and T. Ugajin, Modular Hamiltonians of excited states, OPE blocks and emergent bulk fields, JHEP 01 (2018) 012 [arXiv:1705.01486] [INSPIRE].

[12] S. Hollands and R.M. Wald, Stability of Black Holes and Black Branes, Commun. Math. Phys. 321 (2013) 629 [arXiv:1201.0463] [InSPIRE].

[13] N. Lashkari and M. Van Raamsdonk, Canonical Energy is Quantum Fisher Information, JHEP 04 (2016) 153 [arXiv: 1508.00897] [INSPIRE].

[14] H. Casini and M. Huerta, Entanglement entropy in free quantum field theory, J. Phys. A 42 (2009) 504007 [arXiv:0905.2562] [INSPIRE].

[15] E. Witten, APS Medal for Exceptional Achievement in Research: Invited article on entanglement properties of quantum field theory, Rev. Mod. Phys. 90 (2018) 045003 [arXiv: 1803.04993] [INSPIRE].

[16] D. Petz, Quasi-entropies for states of a von neumann algebra, Publ. Res. Inst. Math. Sci. 21 (1985) 787.

[17] N. Lashkari, Relative Entropies in Conformal Field Theory, Phys. Rev. Lett. 113 (2014) 051602 [arXiv: 1404.3216] [INSPIRE].

[18] K.P. Seshadreesan, L. Lami and M.M. Wilde, Rényi relative entropies of quantum Gaussian states, J. Math. Phys. 59 (2018) 072204 [arXiv:1706.09885] [INSPIRE].

[19] H. Casini, R. Medina, I. Salazar Landea and G. Torroba, Renyi relative entropies and renormalization group flows, JHEP 09 (2018) 166 [arXiv:1807.03305] [INSPIRE].

[20] N. Lashkari, Constraining Quantum Fields using Modular Theory, JHEP 01 (2019) 059 [arXiv: 1810.09306] [INSPIRE].

[21] A. Bernamonti, F. Galli, R.C. Myers and J. Oppenheim, Holographic second laws of black hole thermodynamics, JHEP 07 (2018) 111 [arXiv: 1803.03633] [INSPIRE].

[22] A. May and E. Hijano, The holographic entropy zoo, JHEP 10 (2018) 036 [arXiv: 1806.06077] [INSPIRE].

[23] D.D. Blanco, H. Casini, L.-Y. Hung and R.C. Myers, Relative Entropy and Holography, JHEP 08 (2013) 060 [arXiv:1305.3182] [INSPIRE].

[24] G. Sárosi and T. Ugajin, Relative entropy of excited states in two dimensional conformal field theories, JHEP 07 (2016) 114 [arXiv:1603.03057] [INSPIRE].

[25] G. Sárosi and T. Ugajin, Relative entropy of excited states in conformal field theories of arbitrary dimensions, JHEP 02 (2017) 060 [arXiv:1611.02959] [INSPIRE].

[26] T. Ugajin, Mutual information of excited states and relative entropy of two disjoint subsystems in CFT, JHEP 10 (2017) 184 [arXiv:1611.03163] [INSPIRE].

[27] Y.O. Nakagawa and T. Ugajin, Numerical calculations on the relative entanglement entropy in critical spin chains, J. Stat. Mech. 1709 (2017) 093104 [arXiv: 1705. 07899] [INSPIRE]. 
[28] T. Takayanagi, T. Ugajin and K. Umemoto, Towards an Entanglement Measure for Mixed States in CFTs Based on Relative Entropy, JHEP 10 (2018) 166 [arXiv:1807.09448] [INSPIRE].

[29] N. Lashkari, H. Liu and S. Rajagopal, Modular Flow of Excited States, arXiv:1811.05052 [INSPIRE].

[30] A. Belin, A. Lewkowycz and G. Sárosi, The boundary dual of the bulk symplectic form, Phys. Lett. B 789 (2019) 71 [arXiv: 1806.10144] [INSPIRE].

[31] A. Belin, A. Lewkowycz and G. Sárosi, Complexity and the bulk volume, a new York time story, JHEP 03 (2019) 044 [arXiv: 1811.03097] [INSPIRE].

[32] A. Lewkowycz and J. Maldacena, Generalized gravitational entropy, JHEP 08 (2013) 090 [arXiv: 1304.4926] [INSPIRE].

[33] T. Faulkner, M. Li and H. Wang, A modular toolkit for bulk reconstruction, JHEP 04 (2019) 119 [arXiv: 1806.10560] [INSPIRE].

[34] T. Faulkner and A. Lewkowycz, Bulk locality from modular flow, JHEP 07 (2017) 151 [arXiv: 1704.05464] [INSPIRE].

[35] Y. Chen, X. Dong, A. Lewkowycz and X.-L. Qi, Modular Flow as a Disentangler, JHEP 12 (2018) 083 [arXiv: 1806.09622] [INSPIRE]. 